\title{
The structure of superflat graphs
}

\author{
by
}

\author{
A. A. Ivanov (Wrocław)
}

\begin{abstract}
We prove a structure theorem asserting that each superflat graph is treedecomposable in a very nice way. As a consequence we fully determine the spectrum functions of theories of superflat graphs.
\end{abstract}

0. Introduction. This paper is in a way a continuation of [10], [3], where the investigation of stability of theories of graphs was initiated. In [10], superflat graphs were introduced. In some sense it is possible to consider them as graphs having the simplest theories. For example it was proved in [10] that superflat graphs are stable. So, it is very natural to expect they have nice further properties. The main result of our paper (Theorem 3.4) confirms this. It gives a description of uncountable superflat graphs as trees of models very similar to the structure theorem for $\omega$-stable theories. It generalizes the author's result for ultraflat graphs (see the definition in [3]) which was announced at the Mal'cev Conference in Novosibirsk in 1989. The proof of Theorem 3.4 relies on the analysis of forking and orthogonality of types in superflat graphs, carried out in Section 2 .

The natural application of Theorem 3.4-the description of the spectrum functions of theories of superflat graphs - is given in Section 5. The argument in Section 5 consists in adaptation to the present context of the methods of [12]. [12] deals with $\omega$-stable theories. So in Section 5 we have to consider some additional (not $\omega$-stable) cases. This requires some non-trivial facts on the existence of many pairwise orthogonal types. These facts are proved in Section 4.

Our notation follows [1], [6], [12], [13].

I would like to express my gratitude to the referee for his heroic work with the first version of the paper. Also I am deeply indebted to Ludomir Newelski for a lot of helpful remarks and simplifications (especially in Section 4). 
1. Preliminaries. In this section we introduce the basic notions used in this paper. In Theorem 1.4 we prove that every complete theory of superflat graphs is monadically stable. In fact, the proof is the same as that of Theorem 3 of [3]. In the proof of Theorem 1.4 we use a technical Lemma 1.3. This lemma is an important fact, often used in this paper.

A graph is a structure $\mathfrak{U}=\langle A, R\rangle$ where $R$ is a binary relation on $A$. We say that elements $a, b$ are neighbours ( $a$ and $b$ are connected by an edge) if $\mathfrak{U} \vDash R(a, b) \vee R(b, a)$. A sequence $\left\langle c_{0}, c_{1}, \ldots, c_{t}\right\rangle$ is a path of length $t$ from $c_{0}$ to $c_{t}$ if for all $i<t, c_{i}$ and $c_{i+1}$ are distinct neighbours. Elements $a, b$ are connected in $\mathfrak{U}$ if there is a path from $a$ to $b$ in $\mathfrak{U}$. A component of $\mathfrak{U}$ is a maximal set of pairwise connected elements in $\mathfrak{U}$. If $a$ and $b$ are connected, then the distance from $a$ to $b$, denoted by $d(a, b)$, is the length of the shortest path from $a$ to $b$. If $a, b$ are not connected, we put $d(a, b)=\infty$. If $a=b$, we put $d(a, b)=0$.

For finite $m, n$, let $K_{n}^{m}$ be the class of graphs which can be constructed from the complete graph on $n$ vertices by inserting at most $m$ vertices on each edge. Let $\mathfrak{U}^{*}$ be $\left\langle A, R^{*}\right\rangle$, where

$$
R^{*}=\{(a, b): \mathfrak{U} \vDash R(a, b) \vee R(b, a)\} .
$$

A graph $\mathfrak{U}$ omits $K_{n}^{m}$ if no subgraph of $\mathfrak{U}^{*}$ belongs to $K_{n}^{m}$. $\mathfrak{U}$ is superflat if for each $m, \mathfrak{U}$ omits $K_{n}^{m}$ for some $n$. $\mathfrak{U}$ is ultraflat if there is $n$ such that for all $m$ the graph $\mathfrak{U}$ omits $K_{n}^{m}$.

From now on we assume that $T$ is a complete theory of superflat graphs, and usually all graphs we consider are models of $T$. For convenience we work within a very saturated "monster" model $\mathfrak{C}$ of $T$, that is, we assume that all models of $T$ we consider are elementary submodels of $\mathfrak{C}$ and all sets of parameters are subsets of $\mathfrak{C}$ (of cardinality smaller than the cardinality of $\mathfrak{C})$.

The following lemma is a variant of Lemma 3 from [3].

LEMma 1.1. For each $m \in \omega$ there is a finite $n_{T}(m)$ such that for every algebraically closed set $A$ and $a \notin A$ there are at most $n_{T}(m)$ elements of $A$, connected with a by paths of length $\leq m$ lying outside $A$.

Assume $A$ is algebraically closed, $A \subseteq \mathfrak{N}$ and $a \in \mathfrak{N} \backslash A$. Let $G(a / A)$ (the boundary of $A$ determined by $a$ ) be the set of $b \in A$ connected with $a$ by a path lying outside $A$. By Lemma 1.1, $G(a / A)$ is countable. We say that elements $b_{1}, b_{2} \notin A$ are connected over $A$ if they are connected by a path disjoint from $A . C_{\mathfrak{N}}(a / A)$ (the component of $a$ over $A$ in $\mathfrak{N}$ ) is the set of $b \in \mathfrak{N} \backslash A$ connected with $a$ over $A$. $C(a / A)$ denotes $C_{\mathfrak{C}}(a / A)$.

If $G=G(a / A)$ then clearly $C(a / A)=C(a / G)$, and if $b \in C(a / A)$ then $C(a / A)=C(b / A)$ and $G(a / A)=G(b / A)$. We say also that $C(a / A)$ is a component over $A$ with boundary $G$. 
Lemma 1.2. If $b \in C(a / A)$ then for some $\phi(x)$ over $A \cup\{a\}$,

$$
b \in \phi(\mathfrak{C}) \subseteq C(a / A) .
$$

In particular, $C(a / A)$ is a countable union of $(A \cup\{a\})$-definable sets and $C_{\mathfrak{N}}(a / A)=\mathfrak{N} \cap C(a / A)$.

Proof. Let $n$ be the length of the shortest path connecting $a$ and $b$ outside $A$. By Lemma 1.1, the set

$$
G=\{c \in G(a / A): d(a, c) \leq n\}
$$

is finite. So there is a formula $\phi(x)$ over $A \cup\{a\}$ saying that $x$ is connected with $a$ by a path of length $n$, disjoint from $G$. Clearly $\phi$ satisfies our demands.

Assume $\Gamma, \Gamma^{\prime}$ are components with boundary $G$ (in $\mathfrak{M}, \mathfrak{M}^{\prime}$ respectively). We say that $\Gamma, \Gamma^{\prime}$ are isomorphic over $G$ if the structures $\langle\Gamma \cup G, g\rangle_{g \in G}$ and $\left\langle\Gamma^{\prime} \cup G, g\right\rangle_{g \in G}$ are isomorphic. Similarly we define the notion of elementary equivalence of $\Gamma, \Gamma^{\prime}$ over $G$.

The next lemma slightly generalizes the corresponding results of [7].

Lemma 1.3. (1) Assume for $i=1,2, C_{i} \cup G_{i}$ is a connected superflat graph, $G_{i}$ is a subgraph of a superflat graph $\mathfrak{M}_{i}, \operatorname{card}(G) \leq \omega$, and $C_{i}$ is disjoint from $\mathfrak{M}_{i}$. Suppose $f: G_{1} \rightarrow G_{2}$ is a graph isomorphism,

$$
\begin{aligned}
\left\langle\mathfrak{M}_{1}, g\right\rangle_{g \in G_{1}} & \equiv\left\langle\mathfrak{M}_{2}, f(g)\right\rangle_{g \in G_{1}}, \\
\left\langle C_{1} \cup G_{1}, g\right\rangle_{g \in G_{1}} & \equiv\left\langle C_{2} \cup G_{2}, f(g)\right\rangle_{g \in G_{1}} .
\end{aligned}
$$

Then

$$
\left\langle\mathfrak{M}_{1} \cup C_{1}, g\right\rangle_{g \in G_{1}} \equiv\left\langle\mathfrak{M}_{2} \cup C_{2}, f(g)\right\rangle_{g \in G_{1}} .
$$

(2) In the situation above let $\mathbf{c}_{1}$ and $\mathbf{c}_{2}$ be tuples from $C_{1}$ and $C_{2}$ respectively. Then the condition

$$
\left\langle\mathfrak{M}_{1} \cup C_{1}, \mathbf{c}_{1}, g\right\rangle_{g \in G_{1}} \equiv\left\langle\mathfrak{M}_{2} \cup C_{2}, \mathbf{c}_{2}, f(g)\right\rangle_{g \in G_{1}}
$$

implies

$$
\left\langle C_{1} \cup G_{1}, \mathbf{c}_{1}, g\right\rangle_{g \in G_{1}} \equiv\left\langle C_{2} \cup G_{2}, \mathbf{c}_{2}, f(g)\right\rangle_{g \in G_{1}} .
$$

Proof. (1) Easy.

(2) See the proof of Lemma 3 from [7]. In our case in that proof it is enough to use the function $d_{G}(x, y)$, which is the number of edges in the shortest path from $x$ to $y$ outside $G$, instead of the distance $d(x, y)$.

The following theorem is a variant of Theorem 3 of [2] and the main result of $[10]$.

THEOREM 1.4. $T$ is monadically stable.

Proof. We prove that every expansion $T^{\prime}$ of $T$ by unary predicates is stable. In this expanded language we also have the notions of boundary, component and so on, defined above. Lemmas 1.1-1.3 remain true for $T^{\prime}$. 
Now let $\mathfrak{M}$ be a model of $T^{\prime}$. Suppose $a, b \notin \mathfrak{M}$ and $G(a / \mathfrak{M})=G(b / \mathfrak{M})=G$. If $\operatorname{tp}(a / G)=\operatorname{tp}(b / G)$ then, by Lemma $1.3, \operatorname{tp}(a / \mathfrak{M})=\operatorname{tp}(b / \mathfrak{M})$. This is enough for stability because every boundary is countable.

This theorem has many interesting consequences.

1. By [2] monadic stability is equivalent to tree-decomposability and implies triviality of $T$. So, we conclude that every theory of superflat graphs is trivial and has regular types only.

2. If $T$ is a unidimensional theory of superflat graphs then by [4] it is superstable and by triviality it is $\omega_{1}$-categorical (see [14]).

3. From tree-decomposability and [5] we also deduce that Vaught's conjecture holds for $T$.

4. If $T$ has finite $U$-rank then $T$ is 1-based [9].

2. Independence in superflat graphs. In this section we analyze independence and orthogonality of types in superflat graphs. As a consequence we deduce that no complete theory of superflat graphs has the dimensional order property (Theorem 2.4). Earlier A. Baudisch and the author independently proved this for ultraflat graphs.

The following lemma is similar to the corresponding lemma of [11].

Lemma 2.1. Assume $A=\operatorname{acl}(A) \subseteq B$ and $\mathbf{a} \in \mathfrak{C}$. Then $\operatorname{tp}(\mathbf{a} / B)$ does not fork over $A$ iff for every $a \in \mathbf{a} \backslash A, C(a / A) \cap B=\emptyset$.

Proof. $\Rightarrow$ Suppose $a \in \mathbf{a} \backslash A$ and $C(a / A) \cap B \neq \emptyset$. Let $b \in C(a / A) \cap B$. Thus also $a \in C(b / A)$, and by Lemma 1.2 there is a formula $\phi(x, b)$ over $A \cup\{b\}$ such that $a \in \phi(\mathfrak{C}, b) \subseteq C(b / A)$. We will show that $\phi(x, b)$ forks over $A$. Choose a Morley sequence $b_{i}, i<\omega$, in $\operatorname{stp}(b / A)$. By the proof of Lemma 1.1 (see Lemmas 1, 3 in [3]) we may assume that $C\left(b_{i} / A\right), i<\omega$, are pairwise disjoint. It follows that $\left\{\phi\left(x, b_{i}\right): i<\omega\right\}$ is 2-inconsistent. Indeed, if $a^{\prime}$ realizes $\phi\left(x, b_{i}\right)$ and $\phi\left(x, b_{j}\right)$ then $C\left(b_{i} / A\right)=C\left(a^{\prime} / A\right)=C\left(b_{j} / A\right)$, hence $i=j$. Thus, $\phi(x, b)$ forks over $A$.

$\Leftarrow$ Suppose that for every $a \in \mathbf{a} \backslash A, C(a / A) \cap B=\emptyset$. If $p(\mathbf{x})=\operatorname{tp}(\mathbf{a} / B)$ forks over $A$ then there exists a formula $\phi(\mathbf{x}, \mathbf{b}) \in p(\mathbf{x})$ and a set $\left\{\mathbf{b}_{0}, \mathbf{b}_{1}, \ldots\right\}$ indiscernible over $A$ with $\mathbf{b}=\mathbf{b}_{0}$ such that $\left\{\phi\left(\mathbf{x}, \mathbf{b}_{i}\right): i \in \omega\right\}$ is $k$-inconsistent for some finite $k$.

We can assume that for every $a \in \mathbf{a} \backslash A, C(a / A) \cap \mathbf{b}_{i}=\emptyset$ for each $i$. By Lemma 1.3 we can prove that $\operatorname{tp}(\mathbf{a b} / A)=\operatorname{tp}\left(\mathbf{a b}_{i} / A\right)$ for each $i$. So, $\mathbf{a}$ realizes $\left\{\phi\left(\mathbf{x}, \mathbf{b}_{i}\right): i \in \omega\right\}$. This is a contradiction.

Corollary. Assume $\mathfrak{M}, \mathfrak{N}, \mathfrak{N}^{\prime}$ are models of $T, \mathfrak{M}=\mathfrak{N} \cap \mathfrak{N}^{\prime}$ and all elements $b \in \mathfrak{N} \backslash \mathfrak{M}$ and $b^{\prime} \in \mathfrak{N}^{\prime} \backslash \mathfrak{M}$ are independent over $\mathfrak{M}$. Then $\mathfrak{N} \cup \mathfrak{N}^{\prime}$ is an elementary submodel of $\mathfrak{C}$. 
P r o o f. Suppose $\phi\left(x, \mathbf{b}, \mathbf{b}^{\prime}\right)$ is a formula over $\mathfrak{N} \cup \mathfrak{N}^{\prime}$ with $\mathbf{b} \in \mathfrak{N}, \mathbf{b}^{\prime} \in \mathfrak{N}^{\prime} ;$ we must prove that some $c \in \mathfrak{N} \cup \mathfrak{N}^{\prime}$ realizes $\phi$. Choose any $c$ realizing $\phi$. If $\operatorname{tp}\left(c / \mathbf{b} \mathfrak{N}^{\prime}\right)$ forks over $\mathfrak{N}^{\prime}$ and $\operatorname{tp}\left(c / \mathbf{b}^{\prime} \mathfrak{N}\right)$ forks over $\mathfrak{N}$, then by Lemma 2.1 there is a path disjoint from $\mathfrak{M}$, joining an element of $\mathbf{b}$ to an element of $\mathbf{b}^{\prime}$, a contradiction. So, for example, $\operatorname{tp}\left(c / \mathbf{b} \mathfrak{N}^{\prime}\right)$ does not fork over $\mathfrak{N}^{\prime}$. Hence $\phi\left(x, \mathbf{b}, \mathbf{b}^{\prime}\right)$ does not fork over $\mathfrak{N}^{\prime}$, which means that $\phi\left(x, \mathbf{b}, \mathbf{b}^{\prime}\right)$ is realized in $\mathfrak{N}^{\prime}$.

Now we investigate orthogonality in superflat graphs.

Lemma 2.2. Assume $A \subseteq B, A$ is algebraically closed and $p(\mathbf{x}) \in S(A)$, $q(\mathbf{y}) \in S(B)$. Then $p(\mathbf{x})$ and $q(\mathbf{y})$ are orthogonal iff there are no $\mathbf{a}, \mathbf{b}$ realizing $p, q$ respectively and no component $\Gamma$ over $A$ such that

$$
\Gamma \cap \mathbf{a} \neq \emptyset, \quad \Gamma \cap \mathbf{b} \neq \emptyset, \quad \Gamma \cap B=\emptyset .
$$

Proof. $\Rightarrow$ Suppose a component $\Gamma$ over $A$ meets both $\mathbf{a}$ and $\mathbf{b}$ (for some $\mathbf{a} \vDash p, \mathbf{b} \vDash q)$, and is disjoint from $B$. Let $a \in \Gamma \cap \mathbf{a}, b \in \mathbf{b} \cap \Gamma$. So, $\Gamma=C(a / A)=C(b / A)$, and by Lemma $2.1, \operatorname{tp}(a / B)$ does not fork over $A$. Since $\Gamma \cap B=\emptyset$, we also have $\Gamma=C(b / B)=C(a / B)$. By Lemma 2.1 again, $\operatorname{tp}(a / B \cup\{b\})$ forks over $B$. So, $p$ and $q$ are non-orthogonal.

$\Leftarrow$ A similar proof.

Similarly we get the following lemma.

Lemma 2.3. Assume $A=\operatorname{acl}(A) \subseteq B$ and $\mathbf{a} \in \mathfrak{C}$. Then $q(\mathbf{x})=\operatorname{tp}(\mathbf{a} / B)$ is (almost) orthogonal to $A$ iff for every $a \in \mathbf{a} \backslash A, C(a / A) \cap B \neq \emptyset$.

THEOREM 2.4. $T$ does not have the dimensional order property.

P r o of. Assume $\mathfrak{M}_{0}, \mathfrak{M}_{1}, \mathfrak{M}_{2}$ are models of $T, \mathfrak{M}_{0}=\mathfrak{M}_{1} \cap \mathfrak{M}_{2}$ and $\mathfrak{M}_{1}$, $\mathfrak{M}_{2}$ are independent over $\mathfrak{M}_{0}$. Suppose $\mathfrak{M}_{1}, \mathfrak{M}_{2}$ are $\omega_{\gamma}$-saturated, where $\gamma>0$. It is enough to prove that $\mathfrak{M}_{1} \cup \mathfrak{M}_{2}$ is $\omega_{\gamma}$-saturated (see the corollary of Lemma 2.1). Suppose $A \subseteq \mathfrak{M}_{1} \cup \mathfrak{M}_{2}$ has cardinality $<\omega_{\gamma}$ and $p \in S(A)$ is realized by $a \notin \mathfrak{M}_{1} \cup \mathfrak{M}_{2}$. Consider $G=G\left(a / \mathfrak{M}_{1} \cup \mathfrak{M}_{2}\right)$. By independence of $\mathfrak{M}_{1}, \mathfrak{M}_{2}$ over $\mathfrak{M}_{0}$, using Lemma 2.1 we have $G \subseteq \mathfrak{M}_{1}$ or $G \subseteq \mathfrak{M}_{2}$. Let us consider the first case. Since $G$ is countable, $\operatorname{tp}\left(a / G \cup\left(A \cap \mathfrak{M}_{1}\right)\right)$ is realized in $\mathfrak{M}_{1}$, say by an $a^{\prime}$. By Lemma 1.3 it is easy to see that $a^{\prime}$ realizes $\operatorname{tp}(a / A)$.

3. The structure of superflat graphs. In this section we prove our main theorem asserting that each uncountable superflat graph is treedecomposable in a very nice way.

Assume $p(x) \in S(\mathfrak{U})$ is non-algebraic. We say that $\mathfrak{N}$ is a $p$-model (over $\mathfrak{U})$ if for some $a$ realizing $p, \mathfrak{U} \cup\{a\} \subseteq \mathfrak{N} \subseteq \mathfrak{U} \cup C(a / \mathfrak{U})$. An isomorphism of $p$-models over $\mathfrak{U}$ is called a $p$-isomorphism. The following lemma gives another definition of a $p$-model.

Lemma 3.1. Assume $p=\operatorname{tp}(a / \mathfrak{U})$ and $\mathfrak{U} \cup\{a\} \subseteq \mathfrak{N}$. 
(1) $\mathfrak{N}$ is a p-model iff $\operatorname{tp}(\mathfrak{N} / \mathfrak{U} \cup\{a\})$ is orthogonal to $\mathfrak{U}$.

(2) $\mathfrak{U}^{\prime}=\mathfrak{U} \cup(C(a / \mathfrak{U}) \cap \mathfrak{N})$ is a p-model and an elementary submodel of $\mathfrak{N}$.

Proof. (1) follows quickly from Lemmas 2.1 and 2.3.

(2) Let $\phi(x, \mathbf{b})$ be a formula over $\mathfrak{U}^{\prime}$. We must show that $\phi$ is realized in $\mathfrak{U}^{\prime}$. We may assume $\phi$ is not realized in $\mathfrak{U}$. This means that $\phi$ forks over $\mathfrak{U}$. Let $b \in \mathfrak{N}$ realize $\phi$. By Lemma 2.1, $b \in C(a / \mathfrak{U})$.

If $\mathfrak{U} \cup\{a\} \subseteq B \subseteq \mathfrak{U} \cup C(a / \mathfrak{U})$ and $b \in C(a / \mathfrak{U})$ then $q(y)=\operatorname{tp}(b / B)$ is called a successor of $p(x)=\operatorname{tp}(a / \mathfrak{U})$. Notice that $q(y)$ is orthogonal to $\mathfrak{U}$. If $\mathfrak{U}$ is countable then we define $v(p)$ (the valency of $p$ ) as

$$
\sup \{\operatorname{card}\{q(y) \in S(B): q(y) \text { is a successor of } p(x)\}: \operatorname{card}(B) \leq \omega\} .
$$

The following lemmas use the strategy of $[8]$.

Lemma 3.2. Let $\mathfrak{M}_{0} \cup\{a\} \subseteq \mathfrak{M} \subseteq \mathfrak{N}, p(x)=\operatorname{tp}\left(a / \mathfrak{M}_{0}\right)$ and let $\mathfrak{M}_{0}$ be countable. Assume $\mathfrak{N}$ is a p-model and $v(p) \leq \omega$. Then for some $b \in \mathfrak{N} \backslash \mathfrak{M}$, $\operatorname{tp}(b / \mathfrak{M})$ is strongly regular.

Pr o o f. We have $\mathfrak{N}=\mathfrak{M}_{0} \cup C_{\mathfrak{N}}\left(a / \mathfrak{M}_{0}\right)$. Thus for every $c \in \mathfrak{N} \backslash \mathfrak{M}_{0}$ there is a formula $\psi_{c, \emptyset}(x)$ over $\mathfrak{M}_{0} \cup\{a\}$ such that

(see Lemma 1.2).

$$
c \in \psi_{c, \emptyset}(\mathfrak{C}) \subseteq C\left(a / \mathfrak{M}_{0}\right)
$$

Note that there is a formula $\phi(x, \mathbf{m})$ such that $\mathbf{m} \in \mathfrak{M}, \phi(\mathfrak{M}, \mathbf{m}) \neq$ $\phi(\mathfrak{N}, \mathbf{m})$, and for every formula $\eta\left(x, \mathbf{m}^{\prime}\right)$ over $\mathfrak{M}$, if $\eta\left(\mathfrak{N} \backslash \mathfrak{M}, \mathbf{m}^{\prime}\right) \cap \phi(\mathfrak{N} \backslash \mathfrak{M}, \mathbf{m})$ $\neq \emptyset$ then $\phi(\mathfrak{N} \backslash \mathfrak{M}, \mathbf{m}) \subseteq \eta\left(\mathfrak{N} \backslash \mathfrak{M}, \mathbf{m}^{\prime}\right)$. If not, we can find a tree of formulas $\psi_{\lambda}(x), \lambda \in 2^{<\omega}$, over $\mathfrak{M}$, such that $\psi_{\emptyset}=\psi_{c, \emptyset}$ for some $c \in \mathfrak{N} \backslash \mathfrak{M}$, and for each $\lambda \in 2^{<\omega}, \psi_{\lambda}(\mathfrak{N} \backslash \mathfrak{M}) \neq \emptyset$ and $\psi_{\lambda^{\wedge} 0}(\mathfrak{N} \mid \mathfrak{M}), \psi_{\lambda^{\wedge} 1}(\mathfrak{N} \backslash \mathfrak{M})$ are disjoint subsets of $\psi_{\lambda}(\mathfrak{N} \backslash \mathfrak{M})$. Choose a countable $B$ with $\mathfrak{M}_{0} \cup\{a\} \subseteq B \subseteq \mathfrak{M}$ such that all $\psi_{\lambda}$ 's are over $B$. Thus over $B$ there are $2^{\omega}$ successors of $\operatorname{tp}\left(a / \mathfrak{M}_{0}\right)$, contradicting $v(p) \leq \omega$.

So, we can fix a formula $\phi(x)$ over $\mathfrak{M}$ such that $\phi(\mathfrak{N} \backslash \mathfrak{M}) \neq \emptyset$ and for every $c_{1}, c_{2} \in \phi(\mathfrak{N} \backslash \mathfrak{M})$ the types of $c_{1}$ and $c_{2}$ over $\mathfrak{M}$ are the same. By D.15 of [6], if $b \in \phi(\mathfrak{N} \backslash \mathfrak{M})$ then $\operatorname{tp}(b / \mathfrak{M})$ is strongly regular.

Lemma 3.3. Let $\mathfrak{M}$ be countable, $\mathfrak{M} \cup\{a\} \subseteq B \subseteq \mathfrak{M} \cup C(a / \mathfrak{M})$ and $v(\operatorname{tp}(a / \mathfrak{M})) \leq \omega$. Then there is a prime model over $B$.

Proof. By Lemma 3.1(2), $\mathfrak{N}=\mathfrak{M} \cup C(a / \mathfrak{M})$ is an elementary submodel of $\mathfrak{C}$ and $B \subseteq \mathfrak{N}$. Now $v(\operatorname{tp}(a / \mathfrak{M})) \leq \omega$ implies (as in Lemma 3.2), that there is $\mathfrak{N}^{\prime} \subseteq \mathfrak{N}$ which is constructible over $B$. It follows that $\mathfrak{N}^{\prime}$ is prime over $B$.

In the next theorem we use the following notation: if $\lambda$ is a cardinal and $\delta, \eta \in \lambda^{<\omega}$ then $\delta^{-}=\eta$ means $\delta=\eta^{\wedge}\langle\nu\rangle$ for some $\nu \in \lambda$. 
TheOREM 3.4. Let $\mathfrak{M}$ be a countable submodel of $\mathfrak{N}$ and $\operatorname{card}(\mathfrak{N})=\lambda$. Then there are a tree $I \subseteq \lambda^{<\omega}$ and $\mathfrak{M}_{\eta}, a_{\eta}, \mathfrak{N}_{\eta}$ for $\eta \in I$ satisfying the following conditions:

(1) $\mathfrak{M}_{\emptyset}=\mathfrak{M}, \mathfrak{N}_{\emptyset}=\mathfrak{N}, \mathfrak{N}_{\eta}=\bigcup\left\{\mathfrak{N}_{\sigma}: \sigma^{-}=\eta\right\}, \operatorname{card}\left(\mathfrak{M}_{\eta}\right) \leq \operatorname{card}(\mathfrak{M})$,

(2) if $\eta \neq \emptyset$ then $\mathfrak{M}_{\eta^{-}} \cup\left\{a_{\eta}\right\} \subseteq \mathfrak{M}_{\eta} \subseteq \mathfrak{N}_{\eta} \subseteq \mathfrak{N}_{\eta^{-}}$,

$$
\operatorname{tp}\left(\mathfrak{N}_{\eta} / \mathfrak{M}_{\eta^{-}} \cup\{a\}\right) \perp \mathfrak{M}_{\eta^{-}},
$$

and $\mathfrak{N}_{\eta}$ is the largest set with this condition,

(3) for $\eta \neq \emptyset, p_{\eta}(x)=\operatorname{tp}\left(a_{\eta} / \mathfrak{M}_{\eta^{-}}\right)$is regular,

(4) the set $\left\{\mathfrak{N}_{\sigma}: \eta=\sigma^{-}\right\}$is independent over $\mathfrak{M}_{\eta}$,

(5) if there is $\sigma^{-}$with $\sigma=\eta^{-}$, then $\operatorname{tp}\left(a_{\eta} / \mathfrak{M}_{\eta^{-}}\right) \perp \mathfrak{M}_{\sigma^{-}}$,

(6) if $v\left(p_{\eta}(x)\right) \leq \omega$ then for every $\sigma \geq \eta, \mathfrak{M}_{\sigma}$ is prime over $\mathfrak{M}_{\sigma^{-}} \cup\left\{a_{\sigma}\right\}$,

(7) if $v\left(p_{\eta}(x)\right) \leq \omega, \eta \leq \sigma, \gamma^{-}=\beta^{-}=\sigma$, then $p_{\gamma}(x)$ is strongly regular and the condition $p_{\gamma}(x) \neq p_{\beta}(x)$ implies $p_{\gamma}(x) \perp p_{\beta}(x)$.

Proof. If $\mathfrak{M}_{\eta}$ and $\mathfrak{N}_{\eta}$ are given assume that the set $\left\{a_{\sigma}: \sigma^{-}=\eta\right\}$ is a maximal independent set over $\mathfrak{M}_{\eta}$ in $\mathfrak{N}_{\eta} \backslash \mathfrak{M}_{\eta}$. Let $\mathfrak{N}_{\sigma}=\mathfrak{M}_{\eta} \cup C_{\mathfrak{N}_{\eta}}\left(a_{\sigma} / \mathfrak{M}_{\eta}\right)$. By Lemma 2.1,

$$
\mathfrak{N}_{\eta}=\bigcup\left\{\mathfrak{N}_{\sigma}: \sigma^{-}=\eta\right\}
$$

and the set $\left\{\mathfrak{N}_{\sigma}: \sigma^{-}=\eta\right\}$ is independent over $\mathfrak{M}_{\eta}$. By Lemma 3.1, $\operatorname{tp}\left(\mathfrak{N}_{\sigma} / \mathfrak{M}_{\eta} \cup\left\{a_{\sigma}\right\}\right) \perp \mathfrak{M}_{\eta}$ and $\mathfrak{N}_{\sigma}$ is the largest set with this condition.

Now for every $\sigma$ with $\sigma^{-}=\eta$ choose a countable $\mathfrak{M}_{\sigma} \subseteq \mathfrak{N}_{\sigma}$ containing $\mathfrak{M}_{\eta} \cup\left\{a_{\sigma}\right\}$. It is easy to see that (5) follows by Lemma 2.3 .

To satisfy condition (7) we choose $\left\{a_{\sigma}: \sigma^{-}=\eta\right\} \subseteq \mathfrak{N}_{\eta} \backslash \mathfrak{M}_{\eta}$ as a maximal set independent over $\mathfrak{M}_{\eta}$ whose elements realize strongly regular types over $\mathfrak{M}_{\eta}$. If there is a component $\Gamma$ over $\mathfrak{M}_{\eta}$ (in $\mathfrak{N}_{\eta}$ ) such that $\Gamma \cap\left\{a_{\sigma}: \sigma^{-}=\eta\right\}$ $=\emptyset$, then by Lemmas 3.1 and 3.2, there is an $a \in \Gamma$ realizing a strongly regular type over $\mathfrak{M}_{\eta}$. By Lemma 2.1, $a$ is independent of $\left\{a_{\sigma}: \sigma^{-}=\eta\right\}$. This contradicts the maximality of this set. So, $\mathfrak{N}_{\eta}=\bigcup\left\{\mathfrak{N}_{\sigma}: \sigma^{-}=\eta\right\}$.

If $\operatorname{tp}\left(a_{\gamma} / \mathfrak{M}_{\eta}\right), \operatorname{tp}\left(a_{\beta} / \mathfrak{M}_{\eta}\right)$ are not orthogonal then by Lemma 2.2 they are realized in the same component over $\mathfrak{M}_{\eta}$. By strong regularity any component containing a realization of one of them contains a realization of the other (we use the following fact from [13]: if $p, q \in S(\mathfrak{U}$ ) are not orthogonal and $p$ is strongly regular then every model containing a realization of $q$ contains a realization of $p$ ). This allows us to choose $a_{\beta}$ such that $a_{\gamma}$ and $a_{\beta}$ have the same types over $\mathfrak{M}_{\eta}$.

To satisfy condition (6) it is enough to use Lemma 3.3.

4. The superstable case. From now on we assume that $T$ is superstable not $\omega$-stable. Lemmas 4.1 and 4.2 below assert the existence of many pairwise orthogonal types in some important cases. This will allow us later 
to apply the method of [12] to count the number of models even in the non- $\omega$-stable case.

Lemma 4.1. For some countable algebraically closed set $A, S_{1}(A)$ contains $2^{\omega}$ pairwise orthogonal types.

Proof. Choose a minimal $\alpha$ such that over some algebraically closed countable set $A$ there are $2^{\omega} 1$-types of $\infty$-rank $\alpha$. If the lemma is not true, then for some $p(x) \in S(A), R_{\infty}(p)=\alpha$ and there are uncountably many types $q_{\beta} \in S(A), \beta<\omega_{1}$, of $\infty$-rank $\alpha$ and non-orthogonal to $p(x)$. Let $a$ realize $p(x)$. By Lemma 2.2, for each $\beta, C(a / A)$ contains some $b_{\beta}$ realizing $q_{\beta}$. Let $A^{\prime}=\operatorname{acl}(A \cup\{a\})$. We may assume $b_{\beta} \notin A^{\prime}$ for each $\beta$ ( $A^{\prime}$ is countable). By Lemma $2.1, q_{\beta}^{\prime}=\operatorname{tp}\left(b_{\beta} / A^{\prime}\right)$ forks over $A$, hence $R_{\infty}\left(q_{\beta}^{\prime}\right)<\alpha$. It follows that for some $\alpha^{\prime}<\alpha$, over $A^{\prime}$ there are $2^{\omega}$ types of $\infty$-rank $\alpha^{\prime}$, contradicting the minimality of $\alpha$.

Lemma 4.2. Assume $\mathfrak{U} \cup\{a\} \subseteq B \subseteq \mathfrak{U} \cup C(a / \mathfrak{U})$ and $B$ is countable. If there are uncountably many successors of $p(x)=\operatorname{tp}(a / \mathfrak{U})$ in $S_{1}(B)$, then there is a countable $B^{\prime}$ with $\mathfrak{U} \cup\{a\} \subseteq B^{\prime} \subseteq \mathfrak{U} \cup C(a / \mathfrak{U})$ such that $S_{1}\left(B^{\prime}\right)$ contains $2^{\omega}$ pairwise orthogonal successors of $p(x)$. $B^{\prime}$ may be taken as a p-model.

Proof. Let $\alpha$ be minimal such that there are uncountably many successors (of $p(x)$ ) of $\infty$-rank $\alpha$ over a countable $B^{\prime}$ with

$$
\mathfrak{U} \cup\{a\} \subseteq B^{\prime} \subseteq \mathfrak{U} \cup C(a / \mathfrak{U}) .
$$

By Lemma 1.2 , in fact over $B^{\prime}$ there are $2^{\omega}$ successors of $p(x)$. As in Lemma 4.1 , such a $B^{\prime}$ satisfies our demands. By Lemma 3.1(2), for some countable model $\mathfrak{B}^{\prime}, B^{\prime} \subseteq \mathfrak{B}^{\prime} \subseteq \mathfrak{U} \cup C(a / \mathfrak{U})$. Clearly, $S_{1}\left(\mathfrak{B}^{\prime}\right)$ also contains $2^{\omega}$ orthogonal successors of $p(x)$.

5. The number of uncountable models. In this section all spectrum functions for theories of superflat graphs are described. We use the method of [12], where all spectrum functions for $\omega$-stable theories are found. Really, our proof is not complete. We consider only some exceptional cases. The rest can be checked by adaptation of the proof of Theorem 5.1 in [12] (for example, as in Sections 10, 11 of [8]).

Moreover, it is not difficult to realize every function of [12] in our situation. So, we avoid the $\omega$-stable case. Since unsuperstable theories have the maximum number of models in every uncountable cardinal we consider superstable theories only.

As usual, $I\left(\omega_{\alpha}, T\right)$ denotes the number of models of cardinality $\omega_{\alpha}$. We will use the beth function $\beth_{\gamma}(\kappa)$ (see e.g. [13]).

An element $a \in \mathfrak{C}$ is an infinite point iff $a$ has infinitely many neighbours. 
Lemma 5.1. Assume $T$ is superstable and of depth 1 . Let $I^{*}$ be the set of infinite points.

(1) If $a \notin \operatorname{acl}(\emptyset)$ then $C(a / \operatorname{acl}(\emptyset)) \subseteq \operatorname{acl}(a)$.

(2) $I^{*}$ is finite and $I^{*} \subseteq \operatorname{acl}(\emptyset)$.

(3) If $A=\operatorname{acl}(A)$ and $a \notin A$ then $C(a / A) \subseteq \operatorname{acl}(a)$ and $G(a / A) \subseteq I^{*}$.

(4) $T$ is weakly minimal.

Proof. (1) If $b \in C(a / \operatorname{acl}(\emptyset)) \backslash \operatorname{acl}(a)$, then by Lemma $2.3, \operatorname{tp}(b / a)$ is orthogonal to $\emptyset$, showing that the depth of $\operatorname{stp}(a)$ is $>0$, and so $\operatorname{Dp}(T)>1$.

(2), (3) First notice that since $C(a / A) \subseteq C(a / \operatorname{acl}(\emptyset))$, by (1) we have $C(a / A) \subseteq \operatorname{acl}(a)$. Also, $G(a / A) \subseteq \operatorname{acl}(\emptyset)$. If not, then for any $b \in G(a / A) \backslash$ $\operatorname{acl}(\emptyset), a \in C(b / \operatorname{acl}(\emptyset)) \backslash \operatorname{acl}(b)$, contradicting (1). Similarly we see that no $b \notin \operatorname{acl}(\emptyset)$ is an infinite point, hence $I^{*} \subseteq \operatorname{acl}(\emptyset)$. By compactness, $I^{*}$ is finite. Since any neighbour of any $b \in \operatorname{acl}(\emptyset) \backslash I^{*}$ is in $\operatorname{acl}(\emptyset)$, it follows that $G(a / \operatorname{acl}(\emptyset)) \subseteq I^{*}$. Since $G(a / A) \subseteq G(a / \operatorname{acl}(\emptyset))$, also $G(a / A) \subseteq I^{*}$.

(4) follows from (3) and Lemma 2.1.

Proposition 5.2. Let $T$ be non- $\omega$-stable of depth 1 . Then for every uncountable cardinal $\kappa=\omega_{\alpha}, I(\kappa, T)=\min \left(2^{\kappa},|\alpha+1|^{2^{\omega}}\right)$.

Proof. Assume $\operatorname{card}(\mathfrak{N})=\kappa$. By Lemma 5.1 the number of isomorphism types of components over acl $(\emptyset)$ is not greater than $2^{\omega}$. Counting the number of copies of every component we can associate with $\mathfrak{N}$ a function from $2^{\omega}$ to $|\alpha+1+\omega|$. So, $I(\kappa, T) \leq \min \left(2^{\kappa},|\alpha+1|^{2^{\omega}}\right)$, where $\kappa=\omega_{\alpha}$.

Let us prove the inverse inequality. By Lemma 4.1 there is a countable model $\mathfrak{M}$ with an uncountable set $Q \subseteq S_{1}(\mathfrak{M})$ of pairwise orthogonal types. Since $\mathfrak{M}$ has a finite number of boundaries and all boundaries are finite we may suppose that all types of $Q$ have the same boundary in $\mathfrak{M}$ (this boundary is algebraic over $\emptyset$ ) and for distinct $p, q \in Q, p$ is not a copy of $q$ over $\emptyset$. Now we can build $\min \left(2^{\kappa},|\alpha+1|^{2^{\omega}}\right)$ elementary extensions of $\mathfrak{M}$ by using the usual technique of dimensions of pairwise orthogonal types.

This proposition and Corollary 4.8 of [12] describe all spectrum functions of theories of superflat graphs of depth 1 .

From now on we investigate theories of depth $>1$. For a theory $T$ of finite depth suppose $\operatorname{Dpv}(T)$ is a maximal number $n$ such that there are countable models $\mathfrak{M}_{1}, \ldots, \mathfrak{M}_{n}$ and elements $a_{1}, \ldots, a_{n}$ satisfying the following conditions:

(1) $\mathfrak{M}_{i} \cup\left\{a_{i}\right\} \subseteq \mathfrak{M}_{i+1}, \operatorname{tp}\left(\mathfrak{M}_{i+1} / \mathfrak{M}_{i} \cup\left\{a_{i}\right\}\right) \perp \mathfrak{M}_{i}$ and $\operatorname{tp}\left(a_{i+1} / \mathfrak{M}_{i+1}\right) \perp$ $\mathfrak{M}_{i}$ where $1 \leq i<n$,

(2) $v\left(\operatorname{tp}\left(a_{n} / \mathfrak{M}_{n}\right)\right)>\omega$.

When we replace condition (2) by condition $\left(2^{\prime}\right)$ given below we obtain a definition of the number $\operatorname{Dpw}(T)$. 
$\left(2^{\prime}\right) \operatorname{tp}\left(a_{n} / \mathfrak{M}_{n}\right)$ has infinitely many pairwise orthogonal successors.

Theorem 5.3. Let $T$ be superstable non- $\omega$-stable of depth $d$. For finite $d$ let $d_{1}=\operatorname{Dpv}(T)$ and $d_{2}=\operatorname{Dpw}(T)$.

1. If $\omega<d$ and $\kappa=\omega_{\alpha}$ then $I\left(\omega_{\alpha}, T\right)=\min \left(2^{\kappa}, \beth_{d}(|\alpha+\omega|)\right)$.

2. If $1<d<\omega$ and $\kappa=\omega_{\alpha}$ then

$$
\begin{aligned}
& I\left(\omega_{\alpha}, T\right)=\min \left(2^{\kappa}, \beth_{d-1}(|\alpha+\omega|) \beth_{d_{1}}\left(|\alpha+1|^{2^{\omega}}\right) \beth_{d_{2}}\left(|\alpha+1|^{\omega}\right)\right) \quad \text { or } \\
& I\left(\omega_{\alpha}, T\right)=\min \left(2^{\kappa}, \beth_{d-2}\left(|\alpha+\omega|^{|\alpha|}\right) \beth_{d_{1}}\left(|\alpha+1|^{2^{\omega}}\right) \beth_{d_{2}}\left(|\alpha+1|^{\omega}\right)\right) .
\end{aligned}
$$

Proof. Using the lemmas of Sections 3, 4 we can adapt the proof of Theorem 5.1 in [12] for the case of superflat graphs. Minor modifications have to be made in the proof of 5.17 and 5.18 in [12]. We use the following lemma here.

LEMmA 5.4. Let $q(x) \in S(\mathfrak{M})$ be strongly regular of depth $1, \mathfrak{M}$ be countable and $\mathfrak{N}$ be a q-model. If $C$ is a finite subset of $\mathfrak{N} \backslash \mathfrak{M}$ and $r(y)$ is a successor of $q(x)$ over $\mathfrak{M} \cup C$ then for every subset $B$ of $\mathfrak{N} \backslash \mathfrak{M}$ the dimension in $\mathfrak{N}$ of a non-forking extension $r^{\prime}(y)$ of $r(y)$ over $\mathfrak{M} \cup B \cup C$ is not less than $\operatorname{dim}(r(y), \mathfrak{N})-\operatorname{card}(B)$.

Proof. By Section 2 the dimension of $r(y)$ (and $r^{\prime}(y)$ respectively) in $\mathfrak{N}$ is the number of components in $\mathfrak{N}$ over acl $(\mathfrak{M} \cup C)$ (over acl $(\mathfrak{M} \cup C \cup B)$ ) containing realizations of $r(y)\left(r^{\prime}(y)\right)$. It is easy to check that if a component $\Gamma$ over acl $(\mathfrak{M} \cup C)$ does not contain an element of $B$ then $\Gamma$ is a component over $\operatorname{acl}(\mathfrak{M} \cup C \cup B)$. So,

$$
\operatorname{dim}(r(y), \mathfrak{N})-\operatorname{card}(B) \leq \operatorname{dim}\left(r^{\prime}(y), \mathfrak{N}\right) .
$$

Assume that in the situation of this lemma $q(x)$ has only finite number of successors and $r(y)$ has a finite dimension in some $p$-model. By the construction of 5.17 of [12] we can build $|\alpha+\omega| p$-models. By Lemma 5.4 if such models $\mathfrak{N}_{1}$ and $\mathfrak{N}_{2}$ are eventually $q-q-t$-isomorphic (see [12]) then the difference of dimensions of $r(y)$ in $\mathfrak{N}_{1}$ and $\mathfrak{N}_{2}$ is bounded by some fixed number. This is enough for the situations of 5.17, 5.18 in [12].

\section{References}

[1] J. T. Baldwin, Fundamentals of Stability Theory, Springer, New York, 1985.

[2] J. T. Baldwin and S. Shelah, Second-order quantifiers and the complexity of theories, Notre Dame J. Formal Logic 26 (1985), 229-303.

[3] H. Herre, A. H. Mekler and K. Smith, Superstable graphs, Fund. Math. 118 (1983), 75-79.

[4] E. Hrushovski, Unidimensional theories are superstable, Ann. Pure Appl. Logic 50 (1990), 117-138.

[5] L. F. Low, Superstable trivial theories, preprint. 
[6] M. Makkai, A survey of basic stability theory, with particular emphasis on orthogonality and regular types, Israel J. Math. 49 (1984), 181-238.

[7] L. Marcus, The number of countable models of a theory of one unary function, Fund. Math. 108 (1980), 171-181.

[8] E. A. Palyutin and S. S. Starchenko, Horn theories with non-maximal spectra, in: Model Theory and its Applications, Yu. L. Ershov (ed.), Nauka, Novosibirsk, 1988, 108-161.

[9] A. Pillay, Simple superstable theories, in: Classification Theory, J. T. Baldwin (ed.), Lecture Notes in Math. 1292, Springer, Heidelberg, 1987, 247-263.

[10] K. Podewski and M. Ziegler, Stable graphs, Fund. Math. 100 (1978), 101-107.

[11] A. N. Ryaskin, The number of models of complete theories of unars, in: Model Theory and its Applications, Yu. L. Ershov (ed.), Nauka, Novosibirsk, 1988, 162182 (in Russian).

[12] J. Saffe, The number of uncountable models of $\omega$-stable theories, Ann. Pure Appl. Logic 24 (1983), 231-261.

[13] S. Shelah, Classification Theory and the Number of Non-Isomorphic Models, North-Holland, Amsterdam, 1978.

[14] —, On almost categorical theories, in: Classification Theory, J. T. Baldwin (ed.), Lecture Notes in Math. 1292, Springer, Heidelberg, 1987, 498-500.

\section{INSTITUTE OF MATHEMATICS}

WROCEAW UNIVERSITY

PL. GRUNWALDZKI $2 / 4$

50-384 WROCEAW, POLAND 